\title{
Cartographie De La Dynamique Spatio-Temporelle Des Parcours Naturels Des Troupeaux Transhumants Dans Les Communes De Banikoara Et De Karimama Au Bénin (Afrique De L’ouest)
}

\author{
Nourou Toko Issiaka \\ Doctorant en Géographie et Gestion de l’Environnement, Laboratoire de \\ Cartographie (LaCarto), Université d’Abomey-Calavi (UAC) \\ Ousséni Arouna \\ Enseignant-Chercheur, Ecole des Sciences et Techniques du Bâtiment et de \\ la Route (ESTBR), Université Nationale des Sciences, Technologies, \\ Ingénierie et Mathématiques d'Abomey (UNSTIM)

\section{Ismaïla Toko Imorou} \\ Enseignant-Chercheur, Laboratoire de Cartographie (LaCarto)/ \\ Université d'Abomey-Calavi
}

doi: 10.19044/esj.2016.v12n32p251 URL:http://dx.doi.org/10.19044/esj.2016.v12n32p251

Abstract

Cattle breeding in the Districts of Banikoara and Karimama is characterized by semi-nomadic and transhumance with a food system based on the exclusive use of rangelands and crop residues; in this context, the risk of degradation of these rangelands are so high. The aim of this research is to assess the physiognomy changes recorded in these rangelands from 2000 to 2013.The techniques of the remote sensing in particular the classification of the Landsat images ETM+ of 2000 and OLI-TIRS of 2013 were used. The transition matrix was produced using the Intersect function of the software ArcGIS 10.1. In the same way, the programs "PontiusMatrix22" and "Intensity Analysis02" were used to measure the intensity and the speed of land covers units changes.Thus, the surface of riparian forests and the woodlands and savannas woodlands passed respectively from $1.86 \%$ and $12.69 \%$ in 2000 to $1.82 \%$ and $6 \%$ in 2013.Tree and shrub savannas knew a progressive evolution of their surface which passed from $37.55 \%$ in 2000 to $40.51 \%$ in 2013. In addition, the mosaics of fields and fallow which are used as surfaces of pasture in dries season also knew an appreciable increase in their area which passed from $47.02 \%$ in 2000 to $51.73 \%$ in 2013. In total, there was a regression of forest formations in favor of savanna and 
anthropogenic formations.The highest speed changes was recorded on the level of woodlands and savannas woodlands.

Keywords: Mapping, forest formations, changes, North-Benin

\section{Résumé}

L'élevage bovin dans les Communes de Banikoara et de Karimama est caractérisé par le semi-nomadisme et la transhumance avec un système alimentaire basé sur l'utilisation exclusive des parcours naturels et des résidus de récolte. Dans ce contexte, le risque de dégradation de ces parcours est alors élevé. L'objectif de la présente recherche est d'évaluer les changements physionomiques enregistrés dans ces parcours naturels de 2000 à 2013. Les techniques de la télédétection satellitale notamment l'interprétation et la classification des images Landsat ETM+ de 2000 et OLI-TIRS de 2013 ont permis d'analyser les changements intervenus entre ces deux dates. La matrice de transition a été réalisée à l'aide de la fonction Intersect du logiciel ArcGIS 10.1. Les programmes "PontiusMatrix22" et "Intensity Analysis02" ont permis de mesurer l'intensité et la vitesse de changement des unités d'occupation des terres. Ainsi, la superficie des forêts galeries et des forêts claires et savanes boisées est passée respectivement de 1,86 \% et 12,69\% en 2000 à $1,82 \%$ et $6 \%$ en 2013. Les savanes arborées et arbustives ont connu une évolution progressive de leur superficie qui est passée de 37,55 \% en 2000 à 40,51\% en 2013. Les mosaïques de champs et jachères qui servent d'aires de pâturage en saison sèche ont connu aussi une augmentation de leur superficie qui est passée de $47,02 \%$ en 2000 à $50,73 \%$ en 2013. Au total, on a enregistré une régression des formations forestières au profit des formations savanicoles et anthropiques. La plus grande vitesse de changement a été enregistrée au niveau des forêts claires et savanes boisées.

Mots clés : Cartographie, formations forestières, changements, Nord-Bénin

\section{Introduction}

Dans les régions tropicales, l'environnement végétal fait l'objet de pressions permanentes liées à diverses activités (agriculture, coupe de bois et élevage) dont les conséquences prennent une allure catastrophique, surtout pour les écosystèmes fragiles (Djènontin, 2010). En Afrique subsaharienne, l'élevage traditionnel majoritairement extensif et transhumant occupe une place de choix dans l'économie des pays et contribue d'environ 10 à $20 \%$ à leur Produit Intérieur Brut (Faye \& Alary, 2001). Dans ces régions, l'élevage des ruminants se base sur l'utilisation extensive des pâturages naturels (Sinsin \& Saïdou, 1998). Selon Rivière (1977), l'alimentation des ruminants 
dans les pays tropicaux est assurée à plus de 90 \% par les parcours naturels. L'exploitation de ces parcours naturels par les éleveurs est basée sur une adaptation continue guidée par l'offre fourragère potentielle et les besoins des troupeaux. En effet, les facteurs d'influence des parcours naturels exploités par les troupeaux bovins sont les grandes variabilités climatiques (Sinsin, 1993; Fournier, 1996; Wall et al.,1999) et la forte utilisation des terres par les humains. Ces deux facteurs rendent les écosystèmes des parcours naturels plus susceptibles à une dégradation (Parton et al., 1996).

Le Bénin, un pays côtier de l'Afrique de l'Ouest est considéré comme une zone d'accueil des transhumants. Mais ce canevas est faussé dorénavant par le front agricole qui tend à réduire les zones pastorales au profit de la culture de coton (Tamou, 2002). Les Départements de l'Alibori et du Borgou constituent les terres d'acceuil des troupeaux transhumants transfrontaliers. Ils regroupent de vastes étendues de pâturages naturels estimés entre 7 et 8 millions d'hectares (Djènontin, 2010). Ces espaces sont chaque année pris d'assaut par les transhumants venus du Niger, du Burkina Faso et du Nigeria pour assurer une complémentarité écologique et répondre aux besoins en ressources pastorales de leur bétail.

L’élevage dans les Communes de Banikoara et de Karimama est caractérisé par le semi-nomadisme et la transhumance avec un système alimentaire basé sur l'utilisation exclusive des parcours naturels et des résidus de récolte. La surexploitation de ces parcours, l'extension continue des surfaces cultivées et la croissance du cheptel bovin entrainent la dégradation du couvert végétal de la région qui mérite d'être investiguée de façon approfondie. C'est dans cette perspective que la présente recherche a été initiée.

L’objectif visé est d’évaluer les changements physionomiques enregistrés dans les parcours naturels des troupeaux transhumants dans les Communes de Banikoara et de Karimama de 2000 à 2013. Elle se fonde sur l'hypothèse que les formations forestières de ces parcours naturels connaissent une évolution régressive au profit des formations anthropiques.

\section{Matériel et méthodes Milieu d'étude}

Le secteur d'étude regroupe les Communes de Banikoara (4383 km²) et de Karimama $\left(6102 \mathrm{~km}^{2}\right)$. Il est situé au nord-ouest du Département de l'Alibori entre $10^{\circ} 56^{\prime}$ et $12^{\circ} 24^{\prime}$ de latitude Nord et entre $2^{\circ}$ et $3^{\circ} 16^{\prime}$ de longitude Est (Figure 1). Les aires protégées (Parc National et zone cynégétique) occupent 59 \% de ce secteur. Le secteur d'étude est situé dans les districts phytogéographiques Mékrou-Pendjari et Borgou-Nord et globalement dans la région soudanienne (White, 1983; Adomou et al., 2006). Le climat est de type soudanien avec une température moyenne de 
$28^{\circ} \mathrm{C}$. Les précipitations moyennes annuelles sont de l'ordre de $900 \mathrm{~mm}$ à Banikoara et de $600 \mathrm{~mm}$ à Karimama. Il règne dans ces communes un régime pluviométrique unimodal avec une saison pluvieuse de mai à octobre et une saison sèche de novembre à avril. Les sols rencontrés dans ce secteur sont essentiellement des sols ferrugineux tropicaux. La végétation est composée de galeries forestières, de forêts claires et savanes boisées, des savanes arborées et arbustives et des mosaïques de champs et de jachères. Les principales activités économiques menées dans ces Communes sont l'agriculture, l'élevage et la pêche.

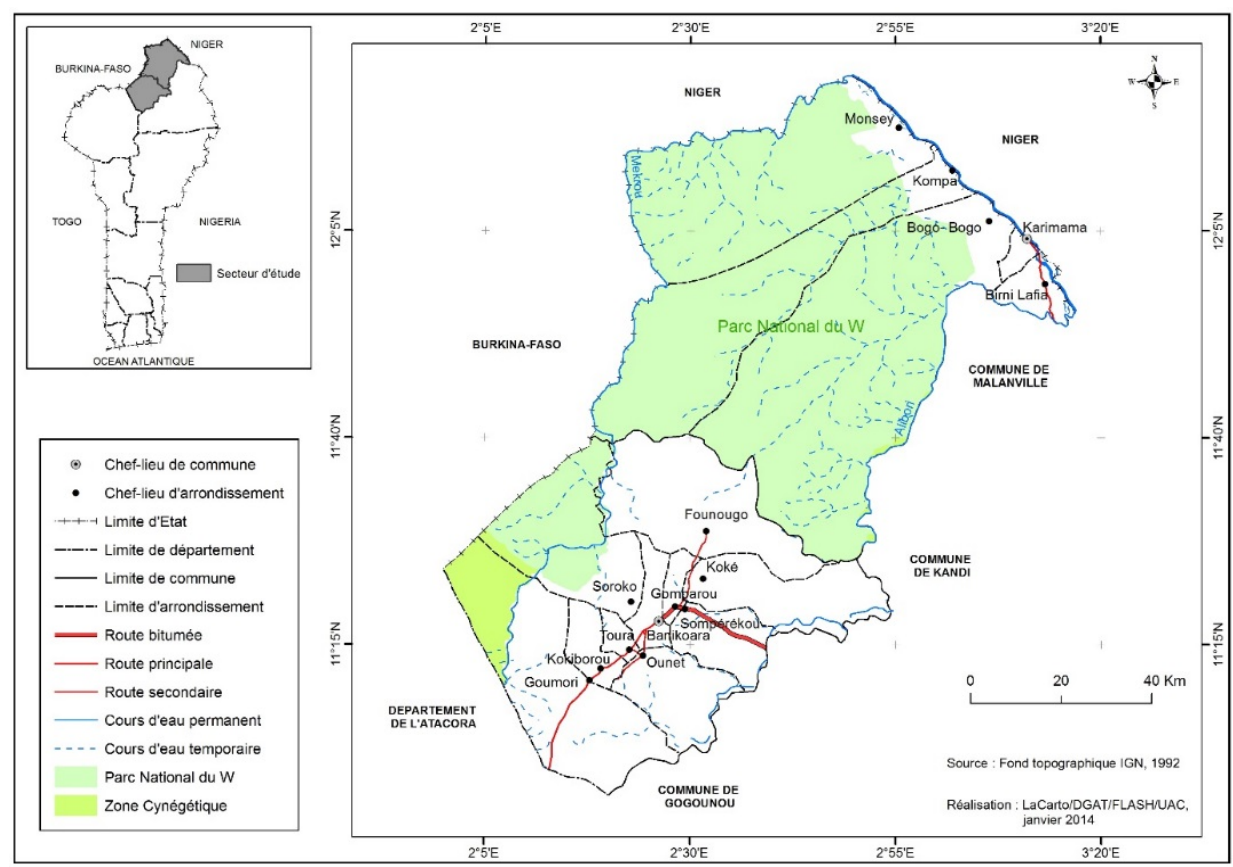

\section{Matériel utilisé}

Figure 1: Situation géographique du secteur d'étude

Dans cette recherche, le matériel utilisé est composé de :

- $\quad$ carte topographique, au 1/200 000, feuille NC-31-XXI de Kandi réalisée par l'Institut Géographique de France, Annexe de Dakar en 1955;

- $\quad$ image Landsat ETM +, scène (P192 et R052) du 26 octobre 2000 ;

- $\quad$ image Landsat OLI/TIRS, même scène (P192 et R052) du 07 novembre 2013.

Ces différentes images ont été téléchargées sur le site de earthexplorer.usgs.gov au format GEOTIFF. Il s'agit des images ayant subi des corrections géométriques (géoréférencement, corrections des distorsions) ;

- $\quad$ logiciel Idrisi Selva 17.0 pour le traitement numérique des images satellitaires Landsat; 
- $\quad$ logiciel ArcGIS 10.1 pour les travaux de cartographie et des analyses SIG ;

- $\quad$ GPS (Global Positioning System) pour le contrôle-terrain ;

- $\quad$ tableur Excel pour le traitement des données statistiques.

Traitement numérique des images satellitaires et cartographie

Cette partie comprend : la composition colorée, le choix des aires d'entrainement et la classification supervisée par maximum de vraisemblance.

\section{Composition colorée}

La composition colorée permet de produire des images en couleurs en tenant compte de la signature spectrale des objets. Elle sert avant tout à distinguer les différents objets présents dans les images afin de faciliter l'interprétation des images. Dans ce traitement, les bandes 4, 5, 3 de Landsat 7 ETM+ et 5, 4, 3 de Landsat 8 ont été respectivement utilisées pour discriminer les différentes unités d'occupation des terres.

\section{Choix des aires d'entraînement}

Les aires d'entraînement sont des sites représentatifs des caractéristiques numériques des classes qui permettent de définir les signatures spectrales de chaque unité paysagère. Les aires d'entraînement ont été délimitées loin des zones de transition afin d'éviter d'inclure des pixels mixtes, c'est-à-dire des pixels qui pourraient être classées dans deux classes distinctes. Sur les images, les aires d'entraînement sont tracées au pixel près et bien dispersées sur l'ensemble du secteur d'étude. Ces aires d'entraînement sont représentatives de la diversité de chaque classe d'unité paysagère. Le nombre d'aires d'entraînement est d'autant plus grand que la classe est hétérogène. La taille de l'aire d'entraînement doit être supérieure à l'erreur de localisation et inférieure à l'objet à détecter (Kioko \& Okello, 2010). Elle a été estimée de la façon suivante : A = P (1+2L) (Arouna, 2012) ; avec $A$ = la superficie de l'aire d'entraînement ; $P$ = dimension du pixel en mètre ; $\mathrm{L}$ = précision de la localisation en mètre.

\section{Classification supervisée par maximum de vraisemblance}

Dans la classification supervisée, l'analyste d'image supervise le processus de catégorisation des pixels en spécifiant à l'algorithme informatique des descripteurs numériques de divers types d'occupation des terres présents dans la scène. Ainsi, des échantillons représentatifs des sites connus (parcelles d'entraînement) ont été utilisés pour établir une caractéristique numérique clé pouvant décrire au mieux les attributs spectraux pour chaque type de classes. Dans ce cas, l'algorithme 
paramétrique choisi est le maximum de vraisemblance. La clé d'interprétation s'est basée sur les points GPS relevés au niveau des différentes unités d'occupation des terres. Au total, six classes communes d’occupation des terres ont été identifiées sur les deux images de 2000 et de 2013. Il s’agit de : savanes arborée et arbustive (SAA); forêt claire et savane boisée (FCSB); forêt galerie (FG); champ et jachère (CJ); plan d'eau (PE) et Agglomération (AG).

Ensuite, le contrôle-terrain a été effectué pour vérifier les classes de pixels issues de la classification.

\section{Analyse statistique des unités d'occupation des terres des parcours naturels \\ Matrice de transition}

La matrice de transition permet de mettre en évidence les différentes formes de conversion qu’ont subies les unités paysagères entre deux années (2000 et 2013). Elle est constituée de $x$ lignes et de $y$ colonnes. Le nombre $x$ de lignes de la matrice indique le nombre d'unités paysagères présentes en 2000 tandis que le nombre $y$ de colonnes de la matrice indique le nombre d'unités paysagères converties en 2013. Quant à la diagonale, elle contient les superficies des unités paysagères restées inchangées. Dans cette matrice, les transformations se font des lignes vers les colonnes. Les superficies de ces différentes classes d'unités paysagères ont été calculées à partir du croisement des cartes d'occupation des terres de 2000 et de 2013 à l'aide de la fonction «Intersect » de l'interface «Arctoolbox » du logiciel ArcGis 10.1 .

\section{Taux de conversion}

Le taux de conversion d'une classe d'occupation des terres correspond au degré de transformation subie par cette classe en se convertissant vers d'autres classes (Arouna, 2012). C'est donc la quantité de changements observés au niveau d'une unité d'occupation du sol entre 2000 et 2013. Il permet ainsi de mesurer le degré de conversion d'une unité d'occupation des terres en d'autres unités. Il est obtenu à partir de la matrice de transition selon la formule suivante (Coulibaly et al., 2016):

$$
T_{c}=\frac{V 1-V 0}{V 0} \times 100
$$

V0: Superficie de l'unité paysagère en 2000 ; V1: Superficie de la même unitéen 2013.

\section{Analyse de l'intensité des changements}

Les programmes d'analyse "PontiusMatrix22" et "Intensity Analysis02.xlms"de Pontius et Schneider (2001) ont été utilisés pour 
mesurer l'intensité et la vitesse des changements des unités d’occupation des terres entre 2000 et 2013.

\section{PontiusMatrix22}

Le programme "PontiusMatrix22"fournit une variété d'analyses et les sommaires statistiques d'une matrice de tableau-croisé (Toko Mouhamadou, 2014). Ce programme a généré deux graphiques en utilisant la matrice de transition entre 2000 et 2013: le premier présente l'intensité des changements survenus au sein de chaque unité d'occupation des terres et le deuxième présente spécifiquement l'intensité de pertes et de gains par unité d'occupation des terres. Au niveau de l'intensité des pertes et des gains, l'état de rapidité des changements est déteminé grâce à la ligne verticale en tiretés, appelée ligne de zone uniforme. Si le graphe est à gauche de cette ligne, le changement est lent ou dormant tandis que si celuici est à droite de cette ligne, le changement est rapide ou actif.

\section{Intensity Analysis02.xlms}

Le programme "Intensity Analysis02.xlms"' grâce à la matrice de transition a permis de générer des statistiques pour les transitions selon les intervalles de temps, entre chaque unité d’occupation des terres.

\section{Résultats}

\section{Etat des formations végétales des parcours naturels et des mosaïques de champs et jachères en 2000 et en 2013}

En 2000, la physionomie de la végétation des parcours naturels était dominée par les savanes arborées et arbustives et les forêts claires et savanes boisées, qui représentaient respectivement $37,55 \%$ et $12,69 \%$ de la superficie (Tableau I, Figure 2,). Les forêts galeries y étaient également rencontrées (1,86 \%) et étaient présentes le long des cours d'eau permanents notamment le Mékrou et l'Alibori. Par ailleurs, les mosaïques de champs et de jachères qui ne sont pas des parcours naturels mais qui constituent des aires de pâturage en saison sèche occupaient 47,02 \% de la superficie.

En 2013, la végétation des parcours naturels du secteur d'étude est dominée par les savanes arborées et arbustives (40,51 \%). On retrouve également les autres formations végétales (forêt claire et savane boisée, forêt galerie) observés en 2000 (Tableau I, Figure 2). 
Tableau I: Superficies des unités d'occupation des terres en 2000 et en 2013

\begin{tabular}{cccccc}
\hline \multirow{2}{*}{ UOT } & \multicolumn{2}{c}{2000} & \multicolumn{2}{c}{2013} & \multirow{2}{*}{ Bilan (\%) } \\
\cline { 2 - 5 } & $\mathrm{S}\left(\mathrm{Km}^{2}\right)$ & $\mathrm{P}(\%)$ & $\mathrm{S}\left(\mathrm{Km}^{2}\right)$ & $\mathrm{P}(\%)$ & \\
\hline FG & 81,38 & 1,86 & 79,31 & 1,82 & $-0,05$ \\
FCSB & 553,81 & 12,69 & 261,29 & 5,99 & $-6,70$ \\
SAA & 1639,07 & 37,55 & 1768,27 & 40,51 & $+2,96$ \\
CJ & 2052 & 47,02 & 2213,93 & 50,73 & $+3,71$ \\
PE & 22,93 & 0,53 & 22,93 & 0,53 & 0 \\
AG & 15,35 & 0,35 & 18,82 & 0,43 & $+0,08$ \\
\hline Total & 4364,54 & 100 & 4364,54 & 100 & \\
\hline
\end{tabular}

Source : Interprétation des images Landsat ETM+ de 2000 et OLI-TIRS de 2013

Légende : UOT : Unité d'occupation des terres ; $\mathrm{S}$ : Superficie ; P : Proportion ; Bilan = $\mathrm{P}_{2013}-\mathrm{P}_{2000}$; FG : Forêt galerie ; FCSB : Forêt claire et savane boisée ; SASA : Savanes arborée et arbustives ; CJ : Mosaïques de champs et jachères ; PE : Plan d'eau ; AG : Agglomération.

De l'examen du tableau I et de la figure 2, il ressort que les forêts galeries, les forêts claires et savanes boisées ont connu une évolution régressive tandis que les savanes arborées et arbustives et les mosaïques de champs et jachères ont connu une évolution progressive.

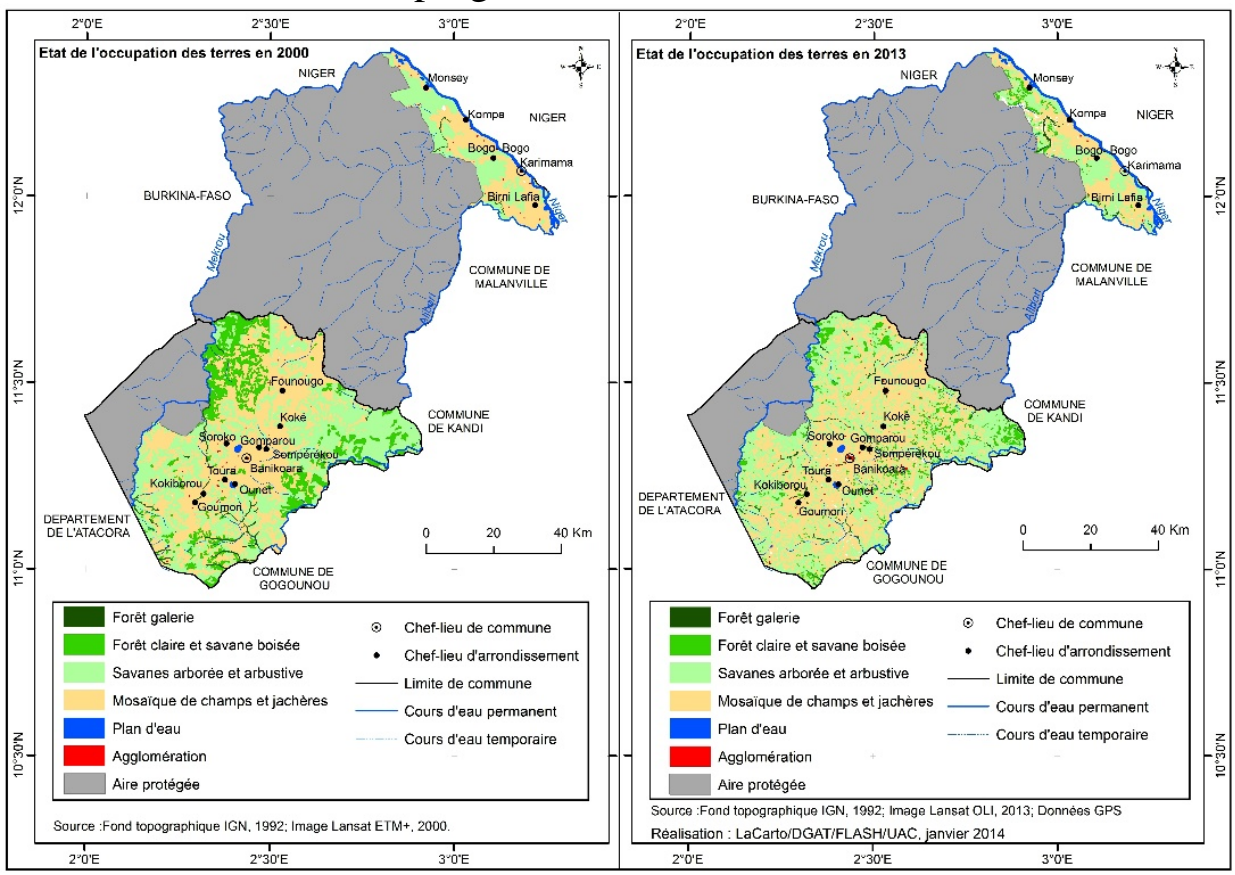

Figure 2 : Unités d’occupation des terres des parcours dans les communes de Banikora et de Karimama en 2000 et en 2013 


\section{Dynamique des formations végétales des parcours naturels et des mosaïques de champs et jachères entre 2000 et 2013}

La dynamique des formations végétales des parcours naturels et des mosaïques de champs et jachères de 2000 à 2013 est résumée par la matrice de transition (Tableau II). Dans les cellules des lignes et des colonnes se trouvent respectivement les unités d'occupation des terres de 2000 et de 2013. Les conversions se font des lignes vers les colonnes. Les cellules de la diagonale correspondent aux superficies des unités d'occupation des terres qui sont demeurées stables de 2000 à 2013. Les unités qui sont en dehors de la diagonale représentent les changements des unités d'occupation des terres.

Tableau II : Matrice de transition de l'occupation des terres entre 2000 et 2013

\begin{tabular}{|c|c|c|c|c|c|c|c|}
\hline \multirow{2}{*}{ Unités de 2000} & \multicolumn{6}{|c|}{ Unités de 2013} & \multirow{2}{*}{ Sup. totale de $2000\left(\mathrm{Km}^{2}\right)$} \\
\hline & FG & FCSB & SAA & $\mathrm{JC}$ & $\mathrm{PE}$ & AG & \\
\hline FG & 79,31 & 0 & 0 & 2,08 & 0 & 0 & 81,38 \\
\hline FCSB & 0 & 249,40 & 112,41 & 192 & 0 & 0 & 553,81 \\
\hline SAA & 0 & 11,89 & 1425,75 & 201,36 & 0 & 0,07 & 1639,07 \\
\hline $\mathrm{CJ}$ & 0 & 0 & 230,11 & 1818,49 & 0 & 3,40 & 2052,00 \\
\hline $\mathrm{PE}$ & 0 & 0 & 0 & 0 & 22,93 & 0 & 22,93 \\
\hline AG & 0 & 0 & 0 & 0 & 0 & 15,35 & 15,35 \\
\hline Sup. totale de $2013\left(\mathrm{Km}^{2}\right)$ & 79,31 & 261,29 & 1768,27 & 2213,93 & 22,93 & 18,82 & 4364,54 \\
\hline
\end{tabular}

Légende : Sup. totale 2000 : Superficie des unités d'occupation du sol en 2000

Sup. totale 2013 : Superficie des autres unités d'occupation du sol en 2013

79,31 : Superficie demeurée stable entre 2000 et 2013

FG : forêt galerie; FCSB : forêt claire et savane boisée ; SASA : savanes arborée et arbustives ; CJ : mosaïques de champs et jachères ; PE : plan d'eau ; AG : agglomération.

L'examen de la matrice de transition (Tableau II) révèle que toutes les unités d'occupation des terres observées en 2000 sont également présentes en 2013. Mais, de façon générale, on remarque que les formations naturelles ont subi deux modes de conversion: la savanisation et l'anthropisation. Les taux de conversion (Tableau III) permettent une meilleure compréhension des différentes transformations subies par la végétation de 2000 à 2013.

Tableau III: Taux de conversion des unités d'occupation des terres entre 2000 à 2013

\begin{tabular}{ll}
\hline Unités d'occupation des terres & Tc (\%) \\
\hline Forêts galeries & 2,55 \\
Forêts claires et savanes boisées & 54,97 \\
Savanes arborées et arbustives & 13,01 \\
Mosaïques de Champs et jachères & 11,38 \\
\hline
\end{tabular}

De l'examen du tableau III, il ressort que le taux de conversion le plus élevé a été enregistré au niveau des forêts claires et savanes arborées avec 54,97 \% ; cela montre que ces unités d'occupation des terres sont fortement perturbées. Par contre, les forêts galeries ont le plus faible taux de conversion $(2,55 \%)$; ce sont des unités d'occupation des terres relativement stables. 


\section{Formes de conversion des formations végétales des parcours naturels}

De 2000 à 2013, les forêts galeries ont connu une diminution de leur superficie qui est passée de $81,38 \mathrm{Km}^{2}$ à $79,31 \mathrm{Km}^{2}$, soit un taux moyen annuel de régression de $0,05 \%$ avec un taux de conversion de 2,55 \%. L'analyse de la matrice de transition montre que 2,08 $\mathrm{Km}^{2}$ des forêts galeries ont été converties en champs et jachères. Les forêts galeries connaissent une évolution régressive au profit des mosaïques de champs et jachères. Cette régression des forêts galeries est due à l'exploitation forestière qui facilite la pratique des activités agricoles dans ces formations.

Les forêts claires et savanes boisées ont également connu une diminution de leur superficie de 2000 à 2013. Elles sont passées de 553,81 $\mathrm{km}^{2}$ à 261,29 $\mathrm{Km}^{2}$ avec un taux annuel de régression de 6,7 \%. L'analyse de la matrice de transition montre que respectivement 112,41 Km² et $192 \mathrm{Km}^{2}$ des forêts claires et savanes boisées ont été converties en savanes arborées et arbustives et en mosaïques de champs et jachères ; soit un taux de conversion de 54,97 \%. Avec cette tendance régressive, c'est une formation végétale qui est en voie de disparition. Cette régression des forêts claires et savanes boisées est due essentiellement aux activités agropastorales et à l'exploitation forestière.

A l'opposé des forêts claires et des savanes boisées, les savanes arborées et arbustives ont connu une évolution progressive de leur superficie de 2000 à 2013 avec un taux annuel de progression de 2,96 \%. En effet, leur superficie est passée de 1639,07 Km² en 2000 à 1768,27 Km² en 2013. Cette tendance est due en grande partie à la conversion des forêts claires et savanes boisées en savanes arborées et arbustives. En effet, l’analyse de la matrice de transition révèle que 112,41 $\mathrm{Km}^{2}$ des forêts claires et savanes boisées ont été transformées en savanes arborées et arbustives. Le taux de conversion qui est de 13,01 \% montre bien une relative stabilité de ces formations végétales de 2000 à 2013.

Par ailleurs les mosaïques de champs et jachères ont connu une extension. Leur superficie est passée de $2052 \mathrm{Km}^{2}$ en 2000 à 2213,93 Km² en 2006. Le taux de progression annuel est de 3,71 \%. Ces mosaïques de champs et jachères constituent des aires de pâturage en saison sèche avec l'utilisation des résidus de récolte.

\section{Synthèse de la conversion des formations végétales des parcours naturels}

Les formations végétales fermées (forêts galeries, forêts claires) des parcours naturels ont connu une diminution de leurs superficies (Figure 3). Une partie des forêts galeries, des forêts claires et savanes boisées s'est transformée en savanes arborées et arbustives et en mosaïques de champs et jachères. Ainsi, les savanes arborées et arbustives sont devenues les 
formations végétales des parcours naturels ayant la plus grande superficie en 2013. On note de façon générale une diminution des superficies des formations végétales naturelles des parcours naturels qui sont passées de 2274,26 Km² en 2000 à 2108,93 $\mathrm{Km}^{2}$ en 2013 au profit des mosaïques de champs et jachères. Cette régression des superficies est le résultat surtout de la pression agricole exercée sur les ressources naturelles dans les Communes de Banikoara et de Karimama. Cette pression agricole est beaucoup plus remarquable dans la Commune de Banikoara qui est la première commune productrice du coton au Bénin.

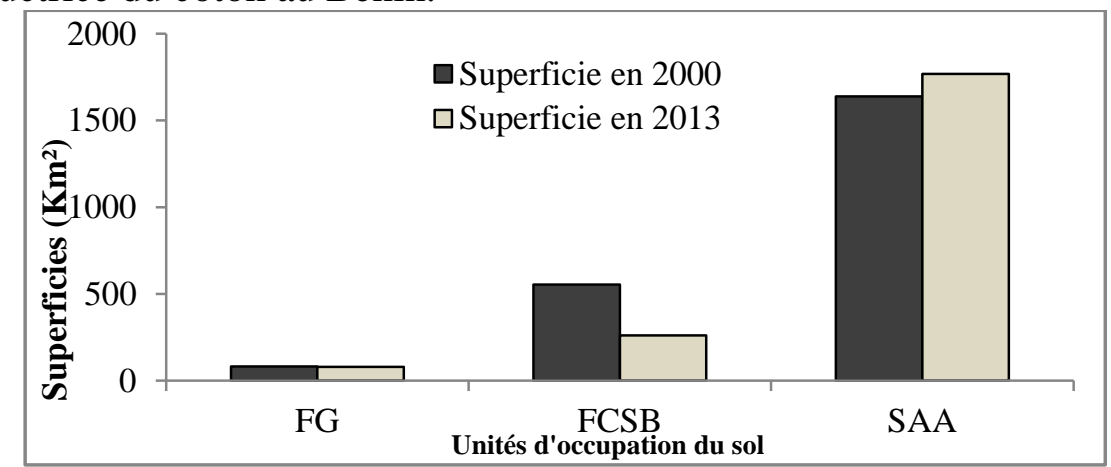

Figure 3: Evolution des formations végétales des parcours naturels entre 2000 et 2013

Légende : FG : forêt galerie ; FCSB : forêt claire et savane boisée ; SAA : savanes arborée et arbustive

De l'examen de la figure 3, il ressort que les régressions ont concerné surtout les forêts galeries, les forêts claires et les savanes boisées qui ont été transformées en savanes arborées et arbustives et en champs et jachères. Ce sont des savanes arborées et arbustives qui ont connu une évolution progressive de leur superficie.

Intensité et vitesse de changements des unités d'occupation des terres Intensité de changements

La figure 4 présente l'intensité des changements entre les unités d'occupation des terres et au sein de chaque unité d'occupation des terres de 2000 à 2013. 


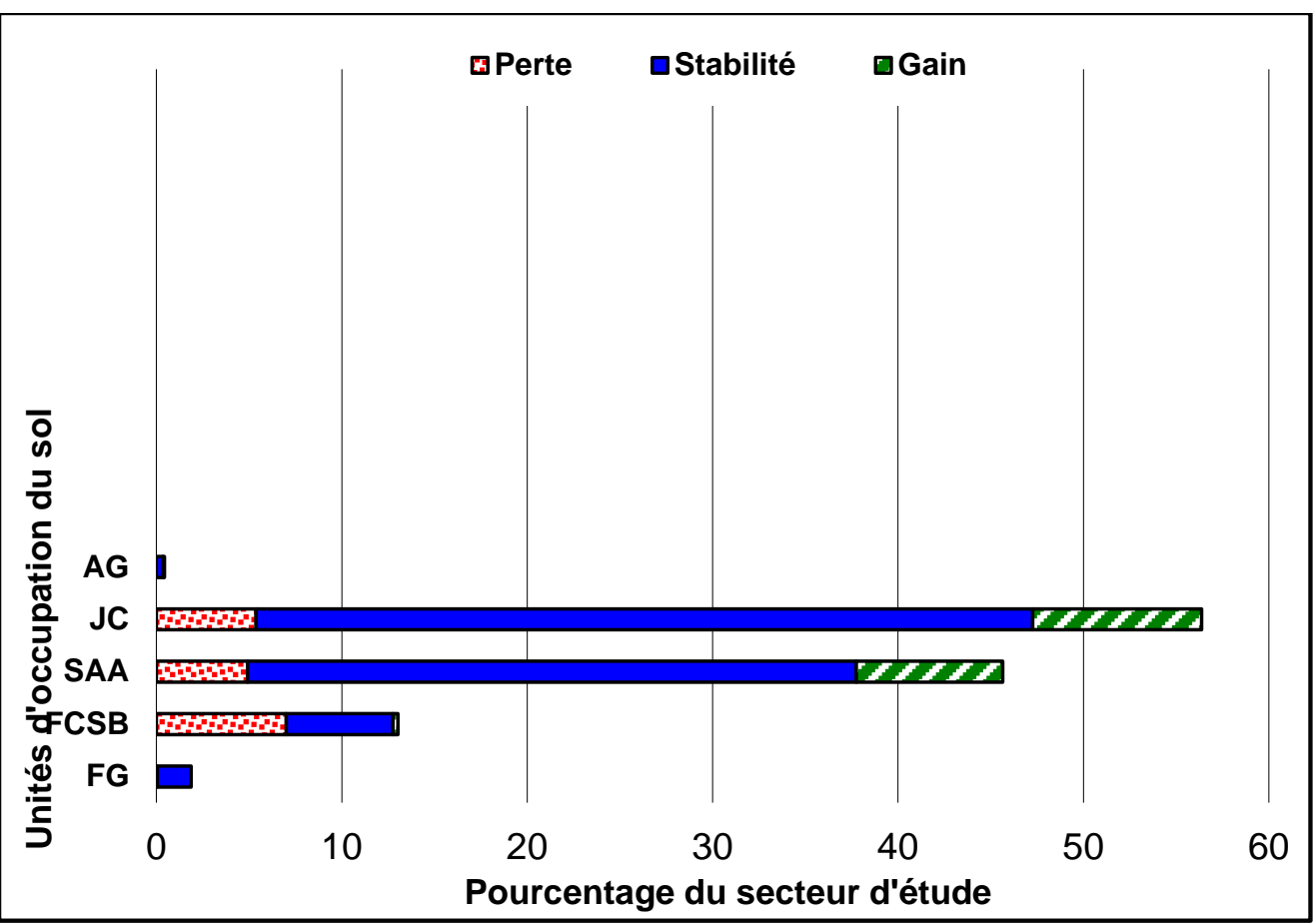

Figure 4:intensité des changements opérés dans les unités d’occupation des terres entre 2000 et 2013

Légende : FG : forêt galerie ; FCSB : forêt claire et savane boisée ; SAA : savanes arborée et arbustive

De l'examen de la figure 4, il ressort de façon générale que les changements entre les unités d'occupation des terres se sont opérés dans le secteur d'étude de façon différentielle sur le plan spatial. Au niveau de chaque unité, on observe des zones de perte, de stabilité ou résistance et de gain occupant chacune des proportions différentes du secteur d'étude. Ainsi, les forêts claires et savanes boisées sont les unités qui ont connu plus de perte (7 \%). Par contre, les savanes arborées et arbustives et les mosaïques de champs et de jachères sont les unités ayant connu plus de gain. Enfin, les forêts galeries sont les seules unités qui ont résisté aux changements notamment la dégradation car elles constituent des écosystèmes protégés par la législation forestière béninoise.

\section{Vitesse de changements des unités d'occupation des terres}

La vitesse de changement à l'intérieur de chaque unité d'occupation des terres entre 2000 et 2013 est déterminée par la ligne verticale en tiretés (intensité uniforme) (Figure 5). 


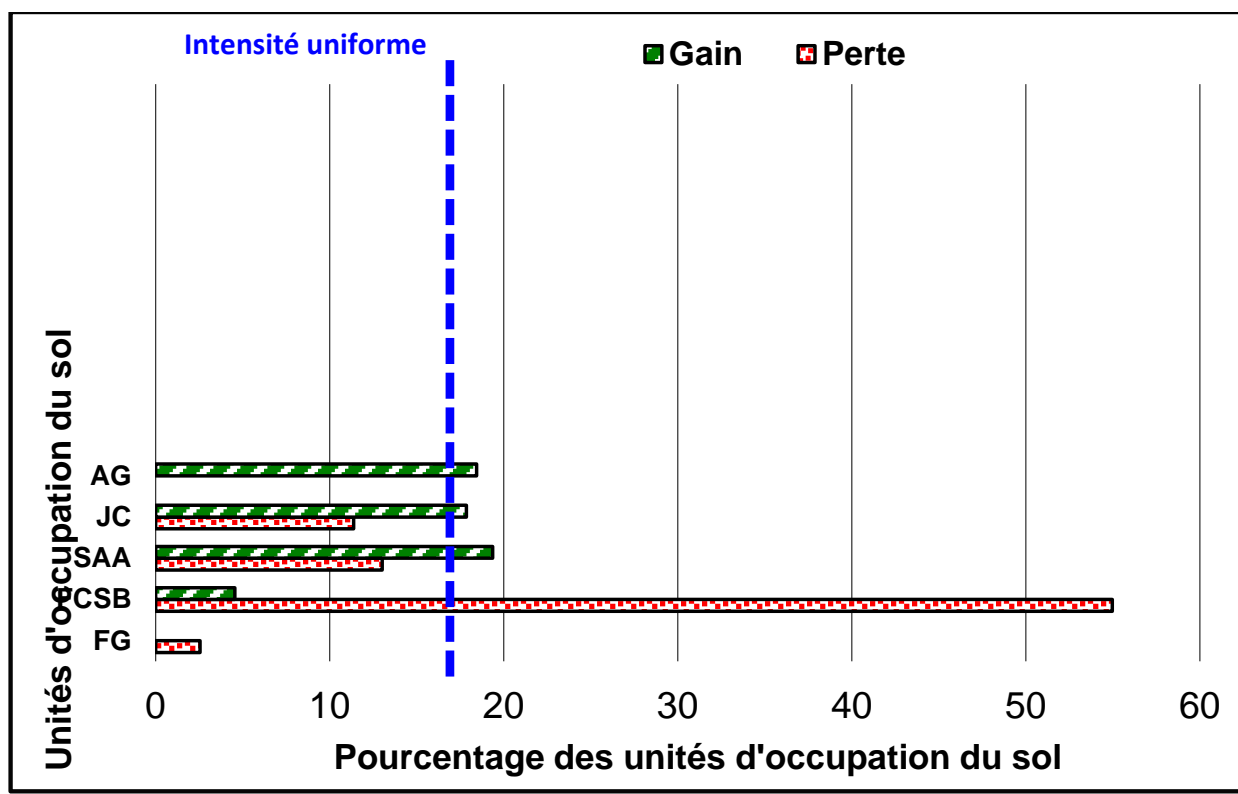

Figure 5: Intensité et vitesse de changement par unité d'occupation des terres entre 2000 et 2013

Légende : FG : forêt galerie ; FCSB : forêt claire et savane boisée ; SAA : savanes arborée et arbustive

L'examen de la figure 5 montre que toutes les unités d'occupation des terres ont connu des changements sous forme de pertes et de gains. Ainsi, en dehors des forêts galeries, les changements opérés au niveau des différentes unités d'occupation des terres du secteur d'étude sont tous actifs ou rapides. La plus grande vitesse de changement a été enregistrée au niveau des forêts claires et savanes boisées avec $55 \%$ de perte et seulement $5 \%$ de gain. Elles sont suivies des savanes arborées et arbustives avec $13 \%$ de perte et $19 \%$ de gain et des mosaïques de champs et jachères avec $11 \%$ de perte et $18 \%$ de gain. Enfin, au niveau des forêts galeries, seules les pertes (3\%) sont observées et la vitesse de changement a été lente. Par contre, on observe uniquement des gains (18\%) au niveau des agglomérations.

\section{Discussion}

\section{Facteurs déterminant la dynamique spatio-temporelle des parcours naturels}

L’approche méthodologique utilisée au cours de cette recherche vient compléter la base de données déjà disponible sur la dynamique de l'occupation des terres. Cette recherche visant à quantifier, localiser et identifier les processus de transition entraînant des changements d'occupation des terres a mis en évidence des dynamiques différenciées pour les différents parcours naturels dans les Communes de Banikoara et de 
Karimama. En effet, les résultats ont révélé que les forêts galeries et les forêts claires et savanes boisées des parcours naturels ont connu une régression de leur superficie en une décennie pratiquement au profit des savanes arborées et arbustives. Les forêts galeries constituent les seules formations forestières qui ont connu très peu de dégradation. En effet, considérant leur unicité, leur vulnérabilité et leur diversité (Natta, 2003), les forêts galeries sont intégralement protégées par la législation forestière béninoise (RB, 1993).

Par ailleurs, les mosaïques de champs et jachères qui servent d'aires de pâturage en saison sèche ont connu une augmentation de leur superficie. Cette tendance régressive des formations fermées au profit des formations ouvertes est due aux pressions agropastorales en général et particulièrement à la culture extensive du coton dans la Commune de Banikoara qui est la première commune productrice du coton au Bénin. Cette extension des champs et jachères concorde avec le fort taux d'accroissement de la population dans ces Communes (Carr et al., 2005; Chazdon et al., 2009).

Plusieurs auteurs sont parvenus à la même conclusion notamment Hountondji (2008), qui a montré que dans les régions soudaniennes et les zones soudano-guinéennes, le taux de diminution moyen annuel de la forêt dense sèche et des forêts galeries qui sont des formations fermées est estimé à 2,8 \%. Cette tendance régressive élevée de cette classe d'occupation des terres s'explique principalement par la culture extensive du coton et les assauts saisonniers des éleveurs transhumants dans les forêts denses sèches et les forêts galeries à la recherche des ressources fourragères et de l'eau. Orékan (2007) a réalisé une projection de la dynamique de l'occupation des terres sur la base des simulations par le modèle Dina-CLUE dans la zone de l’Ouémé Supérieur au Bénin. Ses résultats ont prévu une extension des espaces agricoles au détriment des formations végétales naturelles à l'horizon 2025. Aussi, les travaux de Arouna (2012), ont-ils montré que dans la Commune de Djidja, la dynamique de l'occupation des terres sera davantage caractérisée par la conversion des formations fermées en formations ouvertes. Par ailleurs, il a démontré que les feux de végétation constituent aussi de puissantes armes de destruction massive du couvert végétal notamment des parcours naturels surtout quand ils sont pratiqués de façon tardive. Toko et al. (2010) sont parvenus à la même conclusion en démontrant que la dynamique regressive des formations végétales est essentiellement due à l'agriculture, à l'exploitation forestière, à la carbonisation, à la transhumance et aux feux de végétation. Ces résultats obtenus dans les autres régions du Bénin sont similaires à ceux obtenus dans les Communes de Banikoara et de Karimama. Dans ces deux communes, la pratique des feux de végétation tardifs est l'œuvre des braconniers, des agriculteurs et aussi des éleveurs. Le pâturage aérien est également une 
pratique répandue utilisée par tous les éleveurs pour nourrir leurs troupeaux, surtout en période de soudure. Au total, la dynamique régressive du couvert végétal est imputable majoritairement aux facteurs anthropiques (Scouvart \& Lambin, 2006).

\section{Implications de la dynamique spatio-temporelle des parcours naturels}

La dynamique spatio-temporelle globalement régressive des parcours naturels a des incidences sur le développement des activités agropastorales et sur la biodiversité.

Le broutage et le piétinement modifient la composante herbacée des parcours naturels (Carrière, 1996). Les passages répétés des troupeaux transhumants nationaux et transfrontaliers engendrent une dégradation des sols et du couvert végétal. Il s’ensuit une diminution des ressources fourragères; les performances de l'élevage sont alors compromises. Les mêmes constats ont été faits par Tamou (2002) et Lessé (2011) qui ont montré que le couvert végétal s'éclaircit davantage, la biomasse diminue et les capacités de reproduction et de régénération de la végétation se réduisent de plus en plus affectant ainsi les ressources pastorales.

Le couvert végétal est composé de plusieurs espèces végétales et constitue par ailleurs le milieu-cadre et le milieu-ressource de la faune. La dégradation du couvert végétal a des incidences nuisibles sur la biodiversité. Ainsi, la biodiversité dans les Communes de Banikoara et de Karimama est menacée suite à la dégradation du couvert végétal (Djènontin, 2005 ; Vissin, 2007; Arouna, 2008; Djènontin et al., 2009; Toko et al., 2010; Djohy, 2010 ; Djènontin, 2010 ; Tabou, 2011). C'est une tendance qui rejoint la situation de la plupart des pays de l'Afrique de l'Ouest où la surexploitation des terres est considérée comme la principale cause de la dégradation du couvert végétal et de la perte en biodiversité (Sawadogo et al., 2005; Ozer et al 2007; Bouko et al., 2007 ; Diallo et al., 2011 ).

L'hypothèse de la présente recherche qui stipule que les formations forestières des parcours naturels des Communes de Banikoara et de Karimama connaissent une évolution régressive au profit des formations anthropiques est alors vérifiée. Cette dégradation des formations végétales naturelles a des impacts négatifs sur la biodiversité.

\section{Conclusion}

La présente recherche effectuée sur la cartographie des changements spatio-temporels des parcours naturels dans les Communes de Banikoara et de Karimama a permis d'analyser les changements intervenus dans ces parcours entre 2000 et de 2013. L'analyse cartographique des changements spatio-temporels des parcours naturels a révélé que les formations végétales ont connu de profondes modifications physionomiques dans ces communes. 
Les forêts galeries et surtout les forêts claires et les savanes boisées ont connu une diminution de leur superficie au profit des savanes arborées et arbustives et des mosaïques de champs et jachères.

La dégradation du couvert végétal menace sérieusement la biodiversité. Il importe de prendre des mesures éclairées pour une gestion intégrée des territoires. La gestion intégrée des territoires passera par la prise en compte simultanée des activités humaines, des processus physiques, biologiques et chimiques et des conflits d'intérêt entre les différents usagers concurrents des ressources naturelles en particulier les éleveurs et les agriculteurs. De nouvelles questions complexes se posent alors aux gestionnaires des territoires et aux décideurs. Des outils d'aide à la décision seront alors incontournables pour effectuer des choix éclairés en matière de gestion des territoires dans le but de simuler les évolutions futures de l'occupation des terres afin d'anticiper la dégradation du couvert végétal naturel.

\section{References:}

1. Adomou A.C., Sinsin B. et van der Maesen L. J. G., (2006). Phytosociological and chorological approaches to phytogeography: a meso-scale study in Benin. Syst. Geogr. pl. 76: 155-178

2. Arouna O., (2012). Cartographie et modélisation prédictive des changements spatio-temporels de la végétation dans la Commune de Djidja au Bénin: Implications pour l’aménagement du territoire. Thèse de doctorat en Géographie et Gestion de l'Environnement, Université d’Abomey-Calavi, 246 p.

3. Arouna O., (2008). Evaluation du plan d'aménagement participatif des forêts classées de Goungoun, de la Sota et de la rôneraie de Goroubi au Bénin : détermination des critères et indicateurs locaux de réussite de gestion forestière. Mémoire de maîtrise professionnelle, Université d’Abomey-Calavi, 57 p.

4. Carr L.D., Suter L. \& Barberi A., (2005). Population dynamics and tropical deforestation: state of the debate and conceptual challenges. Population and Environment 27 (1) :89-113.

5. Carrière M., (1996). Impact des systèmes d'élevage pastoraux sur l'environnement en Afrique et en Asie tropicale et sub-tropicale aride et subaride. CIRAD-EMT, 70 p.

6. Chazdon R.L., Harvey C.A., Griffith D.M., Ferguson B. G., Martinez-Ramos M., Morales H., Nigh R., Soto-Pinto L., Breugel M., Philpot S. M., (2009). Beyong reserves: a research agenda for conserving biodiversity in human-modified tropical landscapes. Biotropica, 41 (2):142-153. 
7. Coulibaly l., Kouassi K.H., Soro G. E.\& Savane I., (2016). Analyse du processus de savanisation du nord de la Côte d'Ivoire par télédétection: Cas du département de Ferkessédougou. International Journal of Innovation and Applied Studies, 17 (1), 136-143 p.

8. Djènontin A J., (2005). Interactions élevage-environnement : adaptation des modes d'élevage des bovins à l'extension des espaces cultivés au Nord-Est du Bénin. Mémoire DEA, Université d'Abomey-Calavi, Faculté des Sciences Agronomiques, 112 p.

9. Djènontin A. J., Houinato M., Toutain B. \& Sinsin B., (2009). Pratiques et stratégies des éleveurs face à la réduction de l'offre fourragère au Nord-Est du Bénin. Sécheresse 2009, 20 (4) : pp 34653.

10. Djènontin J. A., (2010). Dynamique des stratégies et des pratiques d'utilisation des parcours naturels pour l'alimentation des troupeaux bovins au Nord-Est du Bénin. Thèse de doctorat, FSA/UAC, 274 p + annexes.

11. Djohy G., (2010). Une analyse des dynamiques sociopolitiques et organisationnelles d'adaptation des éleveurs dans l'Alibori (NordBénin). Mémoire de DEA, FSA, UAC, Abomey-Calavi, Bénin, 128 p.

12. Faye B. \& Alary V., (2001). Les enjeux des productions animales dans les pays du sud. INRA Prod. Anim., 14, pp 3-13. In www.inra.fr

13. Fournier A., (1992). Stratégie de croissance de deux graminées pérennes des savanes soudaniennes d'Afrique de l'Ouest : Andropogon ascinodis et Schizachyrium sanguineum. Muséum National d'Histoire Naturelle, Ecologie générale. Rév. Ecol. (Terre Vie), vol. 47, pp 113- 134.

14. Kioko J. \& Okello M.M., (2010). Land use cover and environmental changes in a semi-arid rangeland, Southern Kenya. Journal of Geography and Regional Planning, 3(11): 322-326.

15. Lessé P., (2011). Analyse de la gestion pastorale et de l'adaptation des éleveurs transhumants face aux variabilités climatiques dans les communes riveraines de la Réserve de Biosphère Transfrontalière du W (Bénin). Mémoire de DEA, FSA/UAC, 85 p.

16. Natta A.K., (2003). Ecological assessment of riparian forests in Bénin: phytodiversity, phytosociology and spatial distribution of trees species. Ph.D Thesis, Wageningen University, 215 p.

17. Parton W. J., Coughenour M. B., Scurlock J. M. O. \& Ojima D. S., (1996). Global grassland ecosystem modeling; development and test of ecosystem models for grassland ecosystems. Scientific Committee on Problems of the Environment (SCOPE), Chichester, NY, E.-U., Wiley, pp. 229-269. 
18. Pontius R.G. \& Schneider L.C., (2001). Land-cover change model validation by an ROC prediction. In Smarandache F., Dezert J.: Advances and Applications of DSmT for information Fusion. From Evidence to Plausible and Paradoxical Reasoning for Land, 62 p.

19. RB (République du Bénin), (1993). Loi n 93-009 du 2 juillet 1993 portant régime des forêts en République du Bénin. DFRN, Cotonou, Bénin, 26 p.

20. Rivière R., (1977). Manuel d'alimentation des ruminants domestiques en milieu tropical. IMVT. Rep. Français Min.de la Coop. 525 p.

21. Scouvart M. \& Lambin E. F, (2006). Approche systémique des causes de la déforestation en Amazonie brésilienne : syndromes, synergies et rétroactions. L’Espace Géographique, 3 : 241-254.

22. Sinsin B. \& Saïdou A., (1998). Impact des feux contrôlés sur la productivité des pâturages soudaniens du nord-est du Bénin. Rev. Elev. Méd. Vét. Pays trop., 42(2) : pp 283-288.

23. Sinsin B., (1993). Phytosociologie, écologie, valeur pastorale, production et capacité de charge des pâturages naturels du périmètre Nikki-Kalalé au Nord du Bénin. Thèse de doctorat, Université Libre de Bruxelles, 390 p.

24. Tabou T., (2011). Aspects socio-économiques et environnementaux de la transhumance dans la Commune de Malanville. Mémoire de maîtrise, FLASH, UAC, Bénin, 90 p.

25. Tamou C., (2002). Etat des lieux spatialisé et quantitatif de la transhumance dans la zone périphérique d'influence du Parc National du W (Bénin). Mémoire d’ingénieur agronome, FSA/UAC, 67 p.

26. Toko I., Arouna O. \& Sinsin B., (2010). Cartographie des changements spatio-temporels de l'occupation du sol dans la forêt classée de l’Alibori supérieur au Nord-Bénin. BenGéo, 7: 22-39.

27. Toko Mouhamadou I., (2014). Facteurs de la fragmentation des écosystèmes forestiers: cas des îlots de forêts denses séches de la forêt classée des Monts Kouffé et de sa périphérie au Bénin. Thèse de Doctorat, Université d’Abomey-Calavi, Bénin, 231 p.

28. Vissin E.W., (2007). Impact de la variabilité climatique et de la dynamique des états de surface sur les écoulements du bassin béninois du Niger. Thèse de doctorat, Université de Bourgogne, $258 \mathrm{p}$.

29. Waal H. O. (de), Combrinck W. J., Howard M. D. \& Fouche H. J., (1999). The impact of climate on veld (natural pasture or rangeland) and animal production. 1990. 283 p.

30. White F., (1983). The vegetation of Africa. A descriptive memoir to accompany the UNESCO/AETFAT/UNSO vegetation map of Africa. UNESCO, Paris, France, 356 p. 